\title{
Experimental Studies of Bose-Einstein Condensates in Disorder
}

\author{
Yong P. Chen ${ }^{\mathrm{a}, *}$, J. Hitchoock ${ }^{\mathrm{b}}$, D. Dries ${ }^{\mathrm{b}}$, S. E. Pollack ${ }^{\mathrm{b}}$ \\ T. A. Corcovilos ${ }^{b}$ and R. G. Hulet ${ }^{b}$ \\ ${ }^{a}$ Department of Physics, Purdue University, 525 Northwestern Ave., West \\ Lafayette, IN 47907, USA \\ ${ }^{\mathrm{b}}$ Department of Physics and Astronomy and Rice Quantum Institute, Rice \\ University, 6100 Main St., Houston TX 77005, USA
}

\begin{abstract}
We review recent studies of the effects of disorder on an atomic Bose-Einstein condensate (BEC). We focus particularly on our own experiments with ${ }^{7} \mathrm{Li}$ BECs in laser speckle. Both the interaction, which gives rise to the nonlinearity in a BEC, and the disorder can be tuned experimentally. This opens many opportunities to study the interplay of interaction and disorder in both condensed matter physics and nonlinear science.
\end{abstract}

Key words: matter wave, Bose-Einstein condensate, interaction, disorder, superfluid

PACS: 03.75.Hh, 03.75.Kk, 64.70.Tg

\section{Introduction}

Since their experimental realizations in 1995, Bose-Einstein condensates (BEC) of ultracold atoms $[1,2]$ have had significant impact on several branches of modern physics. In condensed matter physics, for example, the intrinsic cleanliness coupled with remarkable controllability of physical parameters have made cold atom systems an exceptional venue to study a rich variety of condensed matter problems, ranging from superfluidity to strongly-correlated systems. In particular, cold atoms in optical lattices (periodic potentials) could become a powerful "quantum emulator" for many-body physics (see reviews in [3-5]).

Email address: yongchen@purdue.edu (Yong P. Chen). 
As a coherent matter wave, BEC also provides an extremely interesting system to study in nonlinear science [6]. On a mean-field level, BEC is described by the well-known Gross-Pitaevskii equation (GPE), the time-dependent version of which reads:

$$
i \hbar \frac{\partial \Psi(\vec{r})}{\partial t}=\left(-\frac{\hbar^{2}}{2 m} \nabla^{2}+V(\vec{r})+g|\Psi(\vec{r})|^{2}\right) \Psi(\vec{r})
$$

where $\Psi(\vec{r})$ is the condensate wavefunction, $m$ is the atomic mass, $V(\vec{r})$ is the external potential (for example, the trap potentials that confine the atoms) and $g=4 \pi \hbar^{2} a_{s} / m$ with $a_{s}$ being the $s$-wave scattering length [1]. The GPE is a nonlinear Schrödinger equation [7], with analogous forms appearing in nonlinear optics and many other nonlinear systems. We emphasize that for the BEC, it is the (mean field) interaction $U=g n$, where $n=|\Psi(\vec{r})|^{2}$ being the atomic density, that gives rise to the nonlinear term in (1). Such nonlinearity gives rise to many interesting phenomena in BECs [6], among the most remarkable examples being the observations of various forms of matter wave solitons [8-11].

BECs subjected to disorder (i.e., random potentials) [12] have received great attention in the past few years. Disorder plays a fundamental role in modern condensed matter physics, as most real materials inevitably contain defects, which are often difficult to control. The intrinsic cleanliness of cold atom systems, with the ability to introduce well-controlled disorder, offers unique opportunities to study disordered quantum systems $[13,14]$ and uncover novel quantum phases $[5,15]$. Studying disordered BECs may also provide valuable insights to understand the behaviors of nonlinear systems in the presence of randomness. Since 2005, there have been a flurry of experimental activities [16-27] to study BECs in disordered potentials. The physics investigated have included disorder induced inhibition of transport [16-19,21], effects of disorder on collective modes [16,21,24], Bose glass [20], granular BEC and phase coherence [21], density modulations [21,22], disordered Bose-Hubbard model [23], Anderson localization [25,26] and solitons in disorder [27].

In this proceeding, we review our recent experiments $[21,27]$ on a ${ }^{7} \mathrm{Li} \mathrm{BEC}$ with tunable interaction and subject to a well-controlled disorder made by laser speckle. In the regime of strong repulsive interaction, we have observed that increasing disorder turns the BEC from a superfluid (with expected phase coherence) into an insulating, fragmented "granular" condensate with no global phase coherence. In the regime of moderate attractive interaction, we have performed preliminary studies on the behavior of matter wave solitons in disorder. Our experiments enable studying the interplay between disorder and interaction (or nonlinearity), a topic of great interest in condensed matter and nonlinear physics. 
The rest of the paper is organized as follows: In Sec. 2 and Sec. 3 we review the experimental methods to realize tunable interactions and tunable disorder respectively. These technologies are key for studying the physics discussed in this paper. In Sec. 4, we review the experiments on disordered BECs under various physical conditions, focusing on our results on interacting BECs. The article is concluded in Sec. 5 .

\section{Tunable Interactions}

From Eq. 1 we see that the mean field interaction $U=g n=4 \pi \hbar^{2} n a_{s} / m$ can be controlled by controlling the atom density $(n)$ or the scattering length $a_{s}$. The first method (controlling $n$ ) was used, for example in [25] to reduce the strength of (repulsive) interaction to be sufficiently weak to realize Anderson localization [13]. The second method (controlling $a_{s}$, for example, via a magnetic Feshbach resonance [28] that tunes $a_{s}$ by an external magnetic field $(B))$ is more versatile because both the strength as well as the sign of the interaction can be tuned (from repulsive to attractive). It could in principle reduce the interaction to much smaller strength than what may be practically possible with reducing $n$ (without sacrificing the measurement signal at low $n)$. Several recent experiments [26,27], including ours, have used the Feshbach resonance to tune the interactions.

In our case [21,27], we use the magnetic Feshbach resonance, located near 737 $\mathrm{G}$, in the $\left(F=1, m_{F}=1\right)$ state of ${ }^{7} \mathrm{Li}[9,10,29,30]$. This resonance (Fig. 1$)$ is broad (extending several hundreds of Gauss, alleviating the stringent experimental requirements on controlling and stabilizing the magnetic field), and as a particularly appealing feature, possesses an extremely gradual zero-crossing (in the $B$ dependence of $a_{s}$ ) $[9,10,27,30]$. Using this Feshbach resonance, we can tune the interaction in a wide range to access markedly different regimes, including:

- strongly repulsive, where the BEC wavefunction (in the absence of disorder) is well described by the Thomas-Fermi approximation [1] of the GPE (1);

- (nearly) non-interacting, where the BEC wavefunction is well described by the linear Schrödinger equation;

- moderately attractive: where the BEC can break into bright matter wave solitons $[9,10]$. The solitons are stablized in a quasi-1D trap we use in our experiments.

The remarkable control of the interaction in ${ }^{7} \mathrm{Li}$ is demonstrated in Fig. 1. 


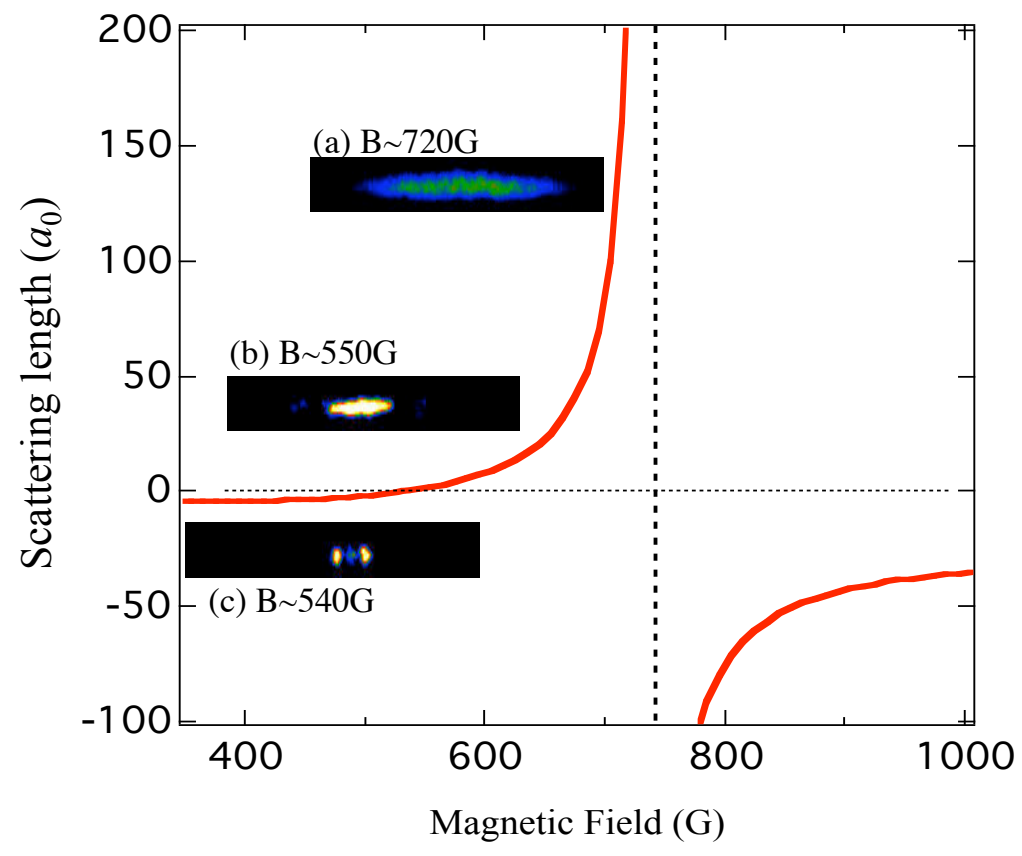

Fig. 1. Calculated scattering length $a_{s}$ (in the unit of Bohr radius $a_{0}$ ) as a function of magnetic field $B$ for ${ }^{7} \mathrm{Li}$ (in the state derived from the $\left(F=1, m_{F}=1\right)$ Zeeman level, which we use in our experiment). The slope of $a_{s}$ vs. $B$ at the zero-crossing is $\sim 0.1$ $a_{0} / \mathrm{G}[30]$. Insets (b)-(c) demonstrate the effect of changing $B$ (thus the interaction) on the BEC: (b) 720G (strongly repulsive); (c) 550G (weakly repulsive); (d) $\sim 540 \mathrm{G}$ (weakly attractive, where solitons are formed $[9,10]$ ). The color scale is individually defined for each inset for clarity.

\section{Tunable Disorder}

Recent experiments on disordered BECs have mostly used various forms of optically generated disorder. In contrast to the disorder due to material defects in typical solid state systems, such optical disorder is of great advantage because the disordered potential is easily tunable and can be precisely characterized. Three types of optical disorder have been used so far: laser speckle $[16,18,21]$, image of a disordered substrate [19,24] and quasi-random (incommensurate) optical lattices [20,26]. The different types of disorder could lead to different physics of disordered BECs, as has been discussed in the case of disorder-induced localization [12,37]. Laser speckle [31], which we use in our experiments, has been the simplest and most popular method to generate optical disorder.

The procedure to create laser speckle in our experiments has been described in 


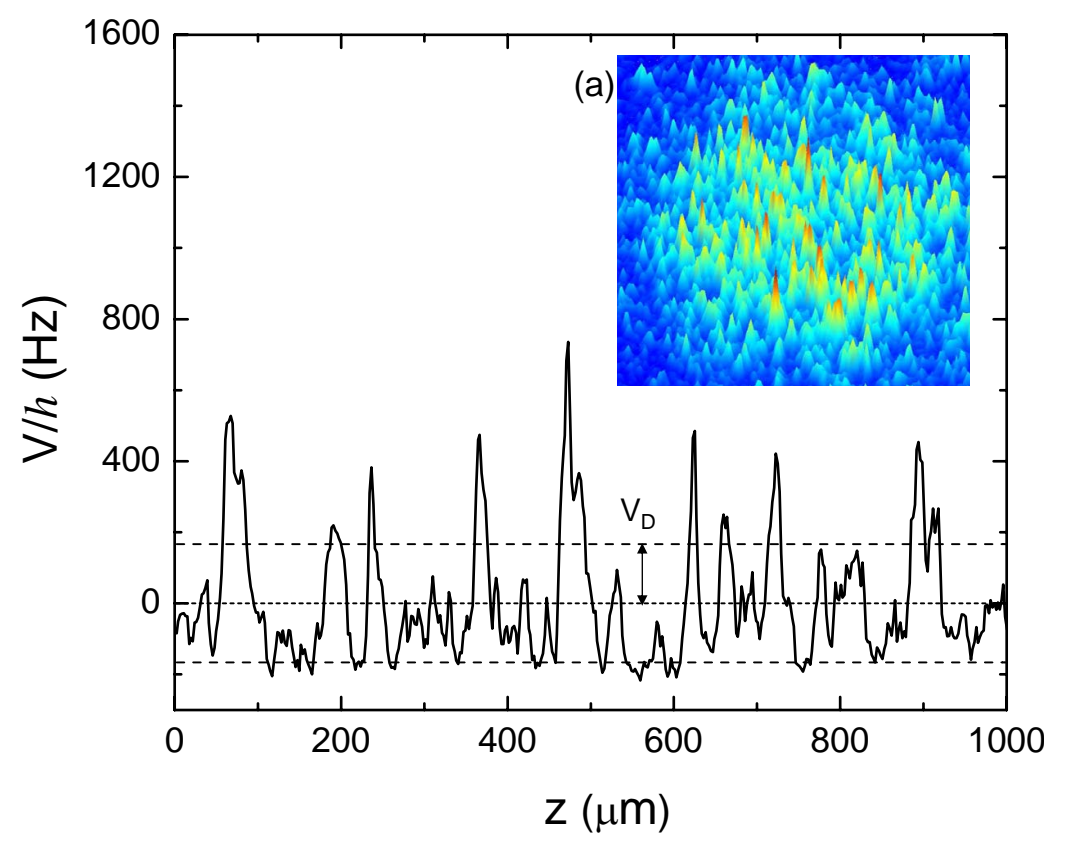

Fig. 2. Typical 1D disordered potential $V(z)$ (adapted from [21]). $V(z)$ is an axial cut $V(0,0, z)$ of the $3 \mathrm{D}$ disordered potential $V(x, y, z)$ through the center $(x=y$ $=0)$ of the atomic cloud. Inset: False-color surface plot of the 2D cross-section $V(0, y, z)$ of the disordered speckle potential as imaged by a camera. The pixel size corresponds to $2.2 \mu \mathrm{m}$ in the plane of the atoms.

detail in [21] and is similar to that used in $[16,18]$. In our case, the BEC, with its small radial size compared to the length scale of the speckle, is to a good approximation subject to a $1 \mathrm{D}$ disordered potential $V(z)$. A representative disordered potential is shown in Fig. 2. The disorder strength, $V_{D}$, proportional to the intensity of the speckle laser, is defined as the standard deviation about the mean of $V(z)$. The disorder correlation length [18,21,31], $\sigma_{z}$, is related to the diffraction limit of the optical system used to generate the speckle [18,31].

\section{Experiments on Disordered BECs}

In this section we review our experiments on interacting ${ }^{7} \mathrm{Li}$ BECs in disorder, focusing on the physics of the interplay between disorder and nonlinearity. A comprehensive review covering many other experiments on disordered BECs can be found in [12]. Discussions on the rich physics related to BECs subject to both disorder and optical lattices, not covered in this article, can be found 
in $[5,20,23]$.

\subsection{Repulsive interaction regime}

We recently reported a systematic study [21] of a repulsively interacting ${ }^{7} \mathrm{Li}$ $\mathrm{BEC}$ in a disordered potential, whose strength $\left(V_{D}\right)$ can be tuned from zero to above the BEC chemical potential $(\mu)$. We have studied both the transport properties and phase coherence of the disordered BEC. We review the experimental observations in this subsection and discuss their physical implications.

\subsubsection{Experimental parameters}

The procedure we use to create a large repulsive ${ }^{7} \mathrm{Li} \mathrm{BEC}$ has been described in detail in [21] and is similar to that used in [10,29]. Except for otherwise specified, the typical parameters of the BEC in our experiments [21] are: scattering length $a_{s} \sim 200 a_{0}$, number of atoms $N \sim 5 \times 10^{5}$, radial trap frequencies $\omega_{x}=\omega_{y} \simeq 2 \pi \times 180 \mathrm{~Hz}$, and the axial trap frequency $\omega_{z} \simeq 2 \pi \times 3.6 \mathrm{~Hz}$. For such a BEC, the chemical potential $\mu \approx h \times 1 \mathrm{kHz}$, where $h$ is Planck's constant. After producing the BEC, the speckle disordered potential $\left(\sigma_{z} \sim 15 \mu \mathrm{m}\right)$ is ramped on to a specific value of $V_{D}$. The BEC is then held in the disordered and trap potentials while various experiments are performed. The final state is probed by absorption imaging of the atomic cloud released from the disordered and optical trap potentials, or by in-situ phase contrast imaging [32]. Both the imaging and speckle lasers propagate along the $x$ axis.

\subsubsection{Transport Properties}

To characterize the transport properties of the disordered BEC, we have carried out two different types of measurements, which we refer to as "dipole oscillation" and "slow drag", with the results summarized in Fig. 3.

In the "dipole oscillation" experiment (Fig. 3a-b), the harmonic trap center is abruptly offset, and the cloud evolves in the presence of the shifted trap and stationary disorder for a variable time before being released for absorption imaging. Without disorder, the condensate undergoes undamped dipole oscillations in the trap with frequency $\omega_{z}$. These oscillations are damped at finite disorder (even as small as $V_{D} \sim 0.1 \mu$ ) and overdamped for $V_{D} \gtrsim$ $0.4 \mu$. The damping coefficient $\beta$ was found to relate to $V_{D}$ by a power law, $\beta \propto\left(V_{D} / \mu\right)^{5 / 2}[27,33]$. When $V_{D} \gtrsim \mu$, the cloud becomes pinned at its initial position to within our experimental resolution.

In the "slow drag" experiment, we slowly (in $\sim 1$ s) ramp on a magnetic field 

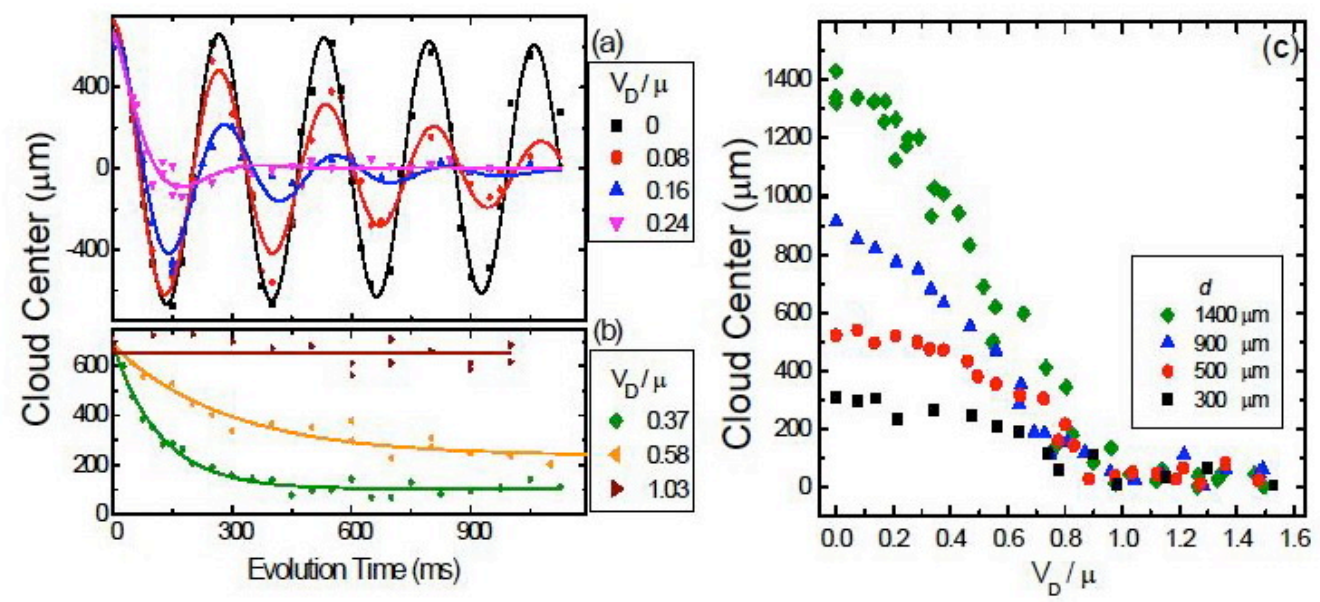

Fig. 3. Effect of disorder on the dipole oscillations (a,b) and slow transport (c) of BEC. See text for details. Figure adapted from [21].

gradient along the $z$ direction, which offsets the center of the harmonic trap from $z=0$ to $z=-d$. The disordered potential is kept stationary during the trap offset. The cloud center as a function of $V_{D}$ for 4 different offset distances $d$ is plotted in Fig. 3c. Without disorder, the center of the atomic cloud follows the trap. For intermediate disorder strength, we have found that the cloud lags behind the new trap center and is stretched [21], indicating inhibited transport. For stronger disorder $\left(V_{D} \gtrsim 0.8 \mu\right)$, the cloud is pinned at its initial position and does not respond to the offset.

\subsubsection{Time of Flight (TOF) Measurements and Phase Coherence}

We have performed both in-situ (phase contrast) and TOF (absorption) imaging on the disordered BEC, yielding information on both its density and phase. Phase contrast imaging [32] is important to obtain accurate in-situ density profiles of the atoms due to the high optical density of the trapped BEC.

Figure 4 compares the in-situ images (a1-a4) and corresponding TOF (taken $8 \mathrm{~ms}$ after releasing the cloud) images (c1-c4) for various $V_{D}$ (from top to bottom, $V_{D} / \mu \approx 0,0.3,0.5$ and 1.0 , respectively). The corresponding axial cuts (column densities), (b1-b4) for in-situ and (d1-d4) for TOF, are also shown. For intermediate $V_{D}$, striking random fringes, which we interpret as matter wave interference, develop after sufficiently long TOF expansion of the BEC following release from the optical potentials (for example, Fig. 4 (c3, d3), where $\left.V_{D} / \mu \sim 0.5\right)$. We note that at such $V_{D}$, the corresponding in-situ images (Fig. 4 (a3, b3)) are consistent with the cloud still being connected. By increasing $V_{D}$ above $\mu$, the disordered BEC becomes fragmented, as shown in the in-situ profile (Fig. 4 (a4,b4)), and the fringe contrast observed in TOF diminishes, as shown in Fig. $4(\mathrm{c} 4, \mathrm{~d} 4)$. Detailed analysis [21] shows that the 

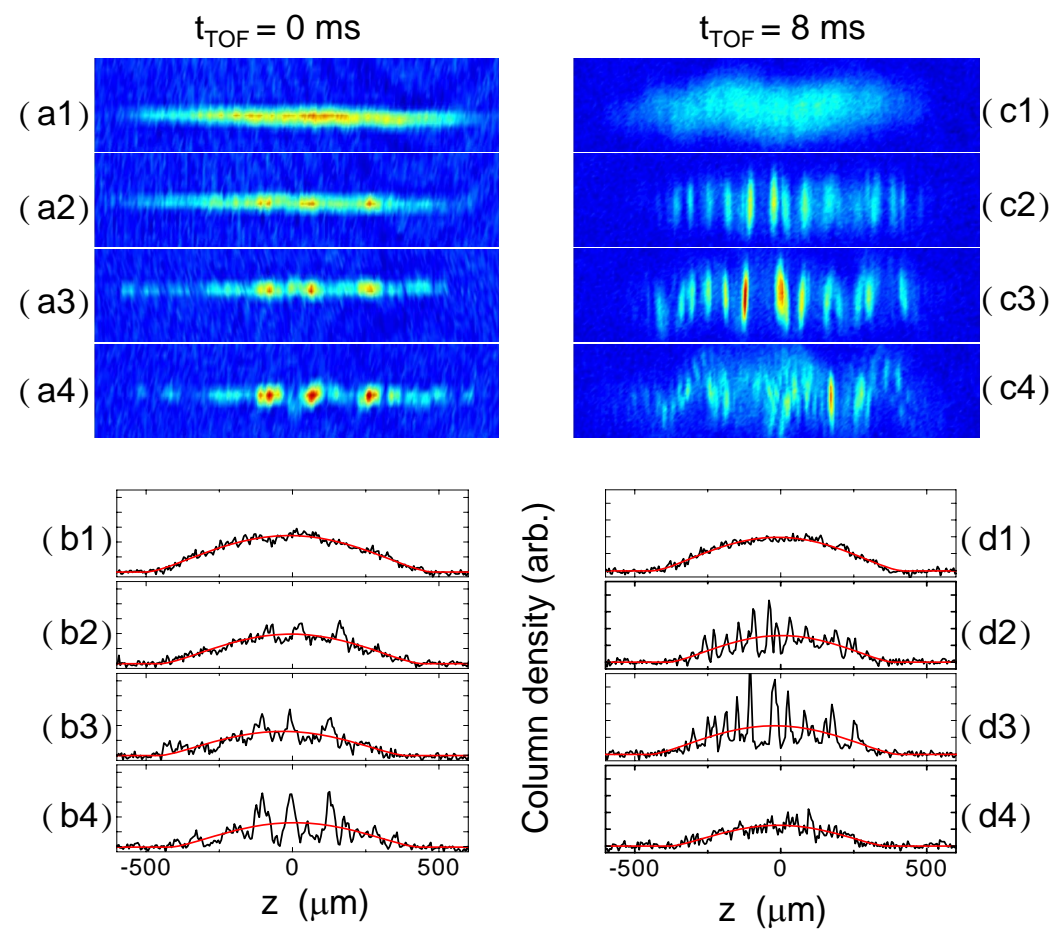

Fig. 4. In-situ profiles and TOF matter wave interference of disordered BEC. (a1-a4) In-situ images for $V_{D} / \mu \approx 0,0.3,0.5$ and 1.0, respectively; (b1-b4) In-situ column-density profiles (axial cuts for images a1-a4); (c1-c4) TOF images taken $8 \mathrm{~ms}$ after releasing the BEC, for $V_{D} / \mu \approx 0,0.3,0.5$ and 1.0, respectively; (d1-d4) TOF column-density profiles (axial cuts for images c1-c4). Solid red lines are fits to Thomas-Fermi distributions. $\omega_{z} \simeq 2 \pi \times 2.8 \mathrm{~Hz}$ for data shown here. The TOF $2 \mathrm{D}$ image uses a different color scale from the one used for the $i n$-situ $2 \mathrm{D}$ image, and the displayed aspect ratio of the in-situ images is reduced by $50 \%$ in order to enhance the transverse size for visibility. Part of figure adapted from [21].

TOF fringe contrast peaks at $V_{D} \simeq 0.5 \mu$, while the contrast in the in-situ images increases up to the highest $V_{D}$ investigated.

\subsubsection{Discussion}

TOF interference fringes probe the phase coherence in the trapped disordered BEC. Similar, but less well resolved fringes in TOF images have been reported previously $[16,19]$. Our highly elongated BEC facilitates a systematic and quantitative study of the fringes. We find the positions of these irregularly spaced fringes are reproducible in repeated measurements, even with different holding times of the BEC in the disordered potential. Another recent experiment [22] has reported reproducible fringes in TOF images of a disordered ${ }^{87} \mathrm{Rb} \mathrm{BEC}$. The reproducibility suggests that these fringes are unlikely due to some initial (before release) phase fluctuations [34] in the disordered BEC, or 
due to interference of a few BECs separated by high potential barriers with no well defined relative phase between them, as in either of these scenarios the fringe positions are not expected to be reproducible. We interpret the reproducible and spatially random density fluctuation we observe in the long time of flight images at intermediate disorder strength as due to the matter wave interference from different parts of the disordered, yet phase coherent, BEC after it is released. This is conceptually analogous with laser speckle, which is the random intensity fluctuation produced by interference from different parts of a phase coherent light (laser) re-scattered from a rough surface. In both cases, phase coherence is crucial for the reproducibility of the resultant density or intensity fluctuation pattern.

Numerical simulations based on the Gross-Pitaevskii equation [19,22] have shown that such interference can occur when moderate disorder-induced density fluctuations cause different parts of a phase coherent BEC to expand with different velocities and overlap. As $V_{D}$ is increased from 0 , the contrast of the interference is expected to increase at small $V_{D}$. When $V_{D}$ becomes sufficiently large, however, the BEC fragments into multiple pieces, as is seen in the insitu image, Fig. 4 (a4,b4). An array of randomly spaced condensates should not produce visible interference in TOF $[35,36]$, again consistent with our observation of diminishing fringe contrast at high $V_{D}$ (Fig. $4(\mathrm{c} 4, \mathrm{~d} 4)$ ). This is quite different from the case of an array of periodically spaced condensates (with negligible tunneling between them), which can still give rise to finite interference (but not reproducible) in a one-shot measurement $[35,36]$.

Regarding the transport properties, we observe completely inhibited transport (pinning of the condensate) at high $V_{D}$, indicating a transition to an insulator. This was seen in previous transport experiments, where the nature of the insulator is inferred to be that of a fragmented BEC [16-18]. Our in-situ images give the first direct observation of fragmentation of a highly disordered BEC. Because of the exponentially suppressed Josephson tunneling between fragments due to high potential barriers, the fragmented BEC is expected to have large phase fluctuations and no phase coherence [37]. This regime is analogous to a granular superconductor $[38,39]$, which is an insulator without global phase coherence.

Dipole oscillations are damped at moderate $V_{D}$, as shown in Fig. 3 (a,b), and in agreement with previous observations [16]. Excitations such as solitons, vortices, and phase slips have been suggested [40] as possible mechanisms giving rise to damping. This is consistent with Landau's criterion for the destruction of superfluidity [1], as we note that at small $V_{D}$, the sound velocity of the BEC at the center of the cloud $c=\sqrt{\mu / m} \sim 8 \mathrm{~mm} / \mathrm{s}$, which is comparable or even smaller than the maximum speed of the moving BEC relative to the static disordered potential (Fig. 3 (a,b)). Our in-situ as well as TOF data at moderate $V_{D}$ suggest a disordered BEC that is still connected (not yet fragmented) and 
phase coherent, in order to give a reproducible interference pattern. Rather than being "granular", this regime is analogous to a "homogeneous" disordered superconductor [38]. Damped dipole oscillations were also recently studied for a BEC in a periodic potential [41] in the context of a possible "Bose metal" phase [42].

Disordered superconductors (either "granular" or "homogeneous" thin films) have been studied extensively [38] in condensed matter physics, in the context of a disorder induced superfluid/supercondutor to insulator transition (SIT), and so-called "dirty boson" problem [14], which is also relevant for superfluid helium in porous media [43] and random Josephson junction arrays [44]. While many believe that such a SIT is a quantum phase transition driven by quantum fluctuations, it remains a central task to understand exactly how the superfluid/superconducting order parameter, which consists of both an amplitude and a phase, may be destroyed with increasing disorder. Our studies on disordered BECs might provide insights into many fundamental questions, such as the nature of the insulator, the form of the order parameter and the fate of phase coherence and phase stiffness [45] throughout the transition, and the possibility of intermediate metallic phases $[38,42,46]$.

\subsection{Attractive interaction regime}

Using the broad Feshbach resonance as described in Sec. 2, one can tune the interaction in the ${ }^{7} \mathrm{Li} \mathrm{BEC}$ from being strongly repulsive, through the zero-crossing (nearly non-interacting), to moderately attractive. Bright matter wave solitons $[9,10]$ can form in such a BEC with attractive interaction. Recently, we have performed similar measurements as described in the previous subsection for such solitons in the presence of speckle disorder. Novel behavior have been observed. For example, the amplitude of the dipole oscillation in moderate disorder can be largely undamped, but the number of atoms continuously decreases during the oscillation [27]. Full results of the on-going experiments on solitons in disorder will be reported in a future publication. We expect such studies to shed new light on the understanding of the interplay between nonlinearity and randomness.

\subsection{Weak interaction regime}

One of the important motivations to study disordered BECs is to observe Anderson localization [13] of matter waves. A necessary condition for Anderson localization $[19,37]$ in a BEC is that the condensate healing length $(\xi=1 / \sqrt{8 \pi n a})$ should be large compared to the disorder correlation length

$\sigma_{z}$. For typical disorder used in the experiments, this condition requires that 
the interaction, or nonlinearity, must be sufficiently weak. Two recent experiments have realized such a weak interaction regime, by either reducing the atomic density [25], or by Feshbach resonance [26], and have directly observed Anderson localization of matter waves.

Using the aforementioned Feshbach resonance in ${ }^{7} \mathrm{Li}$, we have recently realized a regime of extremely weak ( $s$-wave) interaction $\left(a_{s} \lesssim 0.01 a_{0}\right)$ [30]. Such weak interaction provides favorable conditions to observe Anderson localization in disorder. Moreover, we have shown that effects of the anisotropic, long range dipolar interaction (which is normally overwhelmed by the $s$-wave interaction) due to the magnetic moments of ${ }^{7} \mathrm{Li}$ atoms can become observable in this regime [30]. How a dipolar BEC [47] may behave in the presence of disorder is an interesting question that has not yet been addressed.

\section{Conclusion}

In summary, recent experiments, including ours reviewed here, have demonstrated that BECs with tunable interactions and subject to tunable disorder is an exceptional laboratory to study a wide variety of interesting problems in condensed matter physics (such as superfluid-insulator transitions, Anderson localization, Bose glasses) and nonlinear physics (such as solitons in disorder). In particular, understanding the delicate interplay between the interaction (nonlinearity) and randomness will continue to be a key task for current and future investigations in this exciting new field of research.

\section{References}

[1] C. Pethick and H. Smith, Bose-Einstein Condensation in Dilute Gases, 2nd ed., Cambridge (2008)

[2] J. R. Anglin and W. Ketterle, Nature 416, 211 (2002)

[3] I. Bloch, Nature Physics 1, 23 (2005)

[4] I. Bloch, J. Dalibard and W. Zwerger, Rev. Mod. Phys. 80, 885 (2008)

[5] M. Lewenstein, A. Sanpera, V. Ahufinger, B. Damski, A. Sen De and U. Sen, Adv. Phys. 56, 243 (2007)

[6] P. G. Kevrekidis, D. J. Frantzeskakis and R. Carretero-Gonzalez, eds., Emergent Nonlinear Phenomena in Bose-Einstein Condensates, Springer (2008)

[7] C. Sulem and P-L. Sulem, Nonlinear Schrödinger equations: self-focusing and wave collapse, Springer (1991) 
[8] S. Burger et al., Phys. Rev. Lett. 83, 5198 (1999)

[9] L. Khaykovich, F. Schreck, G. Ferrari, T. Bourdel, J. Cubizolles, L. D. Carr,Y. Castin, and C. Salomon, Science 296, 1290 (2002)

[10] K. E. Strecker, G. B. Partridge, A. G. Truscott, and R. G. Hulet, Nature 417, $150(2002)$

[11] B. Eiermann et al., Phys. Rev. Lett. 92, 230401 (2004)

[12] L. Fallani, C. Fort and M. Inguscio, arXiv:0804.2888 (2008), to appear in Adv. Atom Mol. Phys.

[13] P. W. Anderson, Phys. Rev. 109, 1492 (1958); P. A. Lee and T. V. Ramakrishnan, Rev. Mod. Phys. 57, 287 (1985)

[14] M. P. A. Fisher, P. B. Weichman, G. Grinstein, and D. S. Fisher, Phys. Rev. B 40, 546 (1989); W. Krauth, N. Trivedi, and D. Ceperley, Phys. Rev. Lett. 67, 2307 (1991)

[15] B. Damski, J. Zakrzewski, L. Santos, P. Zoller, and M. Lewenstein, Phys. Rev. Lett. 91, 080403 (2003); A. Sanpera, A. Kantian, L. SanchezPalencia, J. Zakrzewski, and M. Lewenstein, Phys. Rev. Lett. 93, 040401 (2004); V. Ahufinger, L. Sanchez-Palencia, A. Kantian, A. Sanpera, M. Lewenstein, Phys. Rev. A 72, 063616 (2005)

[16] J. E. Lye, L. Fallani, M. Modugno, D. S. Wiersma, C. Fort, and M. Inguscio, Phys. Rev. Lett. 95, 070401 (2005)

[17] C. Fort, L. Fallani, V. Guarrera, J. E. Lye, M. Modugno, D. S. Wiersma, and M. Inguscio, Phys. Rev. Lett. 95, 170410 (2005)

[18] D. Clément, A. F. Varòn, M. Hugbart, J. A. Retter, P. Bouyer, L. SanchezPalencia, D. M. Gangardt, G. V. Shlyapnikov, and A. Aspect, Phys. Rev. Lett. 95, 170409 (2005); D. Clément, A. F. Varòn, J. A. Retter, L. Sanchez-Palencia, A. Aspect, and P. Bouyer, New. J. Phys. 8, 165 (2006)

[19] T. Schulte, S. Drenkelforth, J. Kruse, W. Ertmer, J. Arlt, K. Sacha, J. Zakrzewski, and M. Lewenstein, Phys. Rev. Lett. 95, 170411 (2005)

[20] L. Fallani, J. E. Lye, V. Guarrera, C. Fort and M. Inguscio, Phys. Rev. Lett. 98, 130404 (2007); J. E. Lye, L. Fallani, C. Fort, V. Guarrera, M. Modugno, D. S. Wiersma, and M. Inguscio, Phys. Rev. A 75, 061603(R) (2007)

[21] Yong P. Chen, J. Hitchcock, D. Dries, C. Welford and R. G. Hulet, Phys. Rev. A. 77, $033632(2008)$

[22] D. Clément, P. Bouyer, A. Aspect, and L. Sanchez-Palencia, Phys. Rev. A 77, 033631 (2008)

[23] M. White, M. Pasienski, D. McKay, S. Zhou, D. Ceperley and B. DeMarco, arXiv:0807.0446 (2008)

[24] S. Drenkelforth et al., New J. Phys. 10, 045027 (2008) 
[25] J. Billy et al., Nature 453, 891 (2008)

[26] G. Roati et al., Nature 453, 895 (2008)

[27] R. G. Hulet, D. Dries, M. Junker, S. E. Pollack, J. Hitchcock, Y. P. Chen, T. Corcovilos and C. Welford, Proc. Int. Conf. Atom. Mol. Phys. (2008)

[28] S. Inouye, M. R. Andrews, J. Stenger, H. -J. Miesner, D. M. Stamper-Kurn and W. Ketterle, Nature 392, 151 (1998)

[29] M. Junker, D. Dries, C. Welford, J. Hitchcock, Y. P. Chen and R. G. Helet, Phys. Rev. Lett. 101, 060406 (2008)

[30] S. E. Pollack, D. Dries, M. Junker, Y. P. Chen, T. Corcovilos and R. G. Hulet, arXiv:0811.4456 (2008)

[31] J. W. Goodman, Speckle Phenomena in Optics, Roberts (1998)

[32] C. C. Bradley, C. A. Sackett, and R. G. Hulet, Phys. Rev. Lett., 78, 985 (1997)

[33] J. Hitchcock, MS Thesis, Rice University (2008)

[34] S. Dettmer, D. Hellweg, P. Ryytty, J. J. Arlt, W. Ertmer, K. Sengstock, D. S. Petrov, G. V. Shlyapnikov, H. Kreutzmann, L. Santos and M. Lewenstein, Phys. Rev. Lett., 87, 160406 (2001)

[35] Z. Hadzibabic, S. Stock, B. Battelier, V. Bretin, and J. Dalibard, Phys. Rev. Lett. 93, 180403 (2004)

[36] L. Fallani, C. Fort, J. E. Lye, and M. Inguscio, Opt. Express 13, 4303 (2005)

[37] L. Sanchez-Palenci, D. Clément, P. Lugan, P. Bouyer, G. V. Shlyapnikov, and A. Aspect, Phys. Rev. Lett. 98, 210401 (2007); L. Sanchez-Palencia, Phys. Rev. A 74, 053625 (2006); P. Lugan, D. Clément, P. Bouyer, A. Aspect, M. Lewenstein, and L. Sanchez-Palencia, Phys. Rev. Lett. 98, 170403 (2007)

[38] A. M. Goldman and N. Markovic, Phys. Today, Nov, 39 (1998); Y. Dubi, Y. Meir, and Y. Avishai, Nature 449, 876 (2007)

[39] I. S. Beloborodov, A. V. Lopatin, V. M. Vinokur, and K. B. Efetov, Rev. Mod. Phys. 79, 469 (2007)

[40] T. Paul, P. Schlagheck, P. Leboeuf, and N. Pavloff, Phys. Rev. Lett. 98, 210602 (2007)

[41] D. McKay, M. White, M. Pasienski, and B. DeMarco, Nature 453, 76 (2008)

[42] P. Phillips and D. Dalidovich, Science 302, 243 (2003)

[43] J. D. Reppy, J. Low Temp. Phys. 87, 205 (1992)

[44] E. Granato and J. M. Kosterlitz, Phys. Rev. B 33, 6533 (1986); M. Y. Choi, J. S. Chung, and D. Stroud, Phys. Rev. B 35, 1669 (1987)

[45] P. W. Anderson, Basic Notions of Condensed Matter Physics, WestView (1997) 
[46] M. P. A. Fisher, Phys. Rev. Lett. 65, 923 (1990)

[47] T. Koch, T. Lahaye, J. Metz, B. Fröhlich, A. Griesmaier and T. Pfau, Nature Physics 4, 218 (2008) 